\title{
Theranostics
}

Review

2012; 2(1):103-112. doi: 10.7150/thno.3464

\section{Applications of Magnetic Microbubbles for Theranostics}

\author{
Xiaowei Cai, Fang Yang, and Ning Gu ${ }^{凶}$
}

State Key Laboratory of Bioelectronics, Jiangsu Key Laboratory for Biomaterials and Devices, Southeast University, Nanjing, China

Corresponding author: Prof. Dr. Ning Gu, State Key Laboratory of Bioelectronics, Jiangsu Key Laboratory for Biomaterials and Devices, Southeast University, Sipailou 2, Nanjing, China, Tel: +86-(0)25-83272460; e-mail: guning@seu.edu.cn

(c) Ivyspring International Publisher. This is an open-access article distributed under the terms of the Creative Commons License (http://creativecommons.org/ licenses/by-nc-nd/3.0/). Reproduction is permitted for personal, noncommercial use, provided that the article is in whole, unmodified, and properly cited.

Received: 2011.08.31; Accepted: 2011.12.01; Published: 2012.01.12

\begin{abstract}
Compared with other diagnostic methods, ultrasound is proven to be a safe, simple, non-invasive and cost-effective imaging technique, but the resolution is not comparable to that of magnetic resonance imaging (MRI). Contrast-enhanced ultrasound employing microbubbles can gain a better resolution and is now widely used to diagnose a number of diseases in the clinic. For the last decade, microbubbles have been widely used as ultrasound contrast agents, drug delivery systems and nucleic acid transfection tools. However, microbubbles are not fairly stable enough in some conditions and are not well administrated distributed in the circulation system. On the other hand, magnetic nanoparticles, as MRI contrast agents, can non-specifically penetrate into normal tissues because of their relatively small sizes. By taking advantage of these two kinds of agents, the magnetic microbubbles which couple magnetic iron oxides nanoparticles in the microbubble structure have been explored. The stability of microbubbles can be raised by encapsulating magnetic nanoparticles into the bubble shells and with the guidance of magnetic field, magnetic microbubbles can be delivered to regions of interest, and after appropriate ultrasound exposure, the nanoparticles can be released to the desired area while the magnetic microbubbles collapse. In this review, we summarize magnetic microbubbles used in diagnostic and therapeutic fields, and predict the potential applications of magnetic microbubbles in the future.
\end{abstract}

Key words: Magnetic microbubble; Dual-modality imaging; Drug delivery system; Molecular imaging.

\section{Introduction}

Among all the diagnostic imaging techniques, ultrasound imaging has a unique advantage because of its features of real-time, low-cost, high safety, and ease of incorporation into portable devices. By using the ultrasound contrast agents, the resolution and sensitivity of clinical ultrasound imaging have been greatly improved [1-3]. Gas filled microbubbles encapsulated with the polymer, lipid or surfactant shells have been well established for the last decade. Because of their high compressibility, microbubbles can be used as the most effective contrast agent for ultra- sound imaging [4, 5]. Moreover, with the use of sonoporation [6-8], microbubbles are also becoming widely used as drug delivery systems and nucleic acid transfection tool [9-12]. However, the transfection efficiency is limited by ultrasound intensity. As an imaging tool, the imaging quality of ultrasound is suboptimal with contrast lower than that of computer tomography (CT) or magnetic resonance imaging (MRI) [13-16].

On the other hand, MRI is another imaging tool which is non-invasive and capable of providing 
morphological and functional information with a high spatial resolution and excellent soft-tissue contrast [17]. Magnetic nanoparticles can be used as powerful contrast agents for MRI [18, 19]. Especially, magnetic iron oxide nanoparticles with superparamagnetic property have also shown potential as multifunctional nanoparticles for clinical translation besides they have been used as MRI constrast agents in clinic because their features could be easily tailored by including targeting moieties, fluorescence dyes, therapeutic agents or agents. For example, they can also be used as potential anti-cancer agents for their cytotoxicity, genotoxicty and hyperthermia [20-22].

The combination of microbubbles and magnetic nanoparticles, that is, the magnetic microbubbles can make use of the disadvantages of microbubbles or magnetic nanoparticles respectively: the stability of microbubbles can be improved by embedded magnetic nanoparticles into the bubble shells [23], meanwhile, the embedded nanoparticles can be delivered into desired regions with the guidance of magnetic field and can be released when choosing appropriate ultrasound exposure [24, 25]. Because microbubbles are ultrasound contrast agents and magnetic nanoparticles are good contrast agents for MRI, magnetic microbubbles also can potentially be used as contrast agents for both ultrasound imaging and MRI [17, 23, 26 , 27]. With the help of magnetic force, magnetic microbubbles with specific targeting molecules can bind

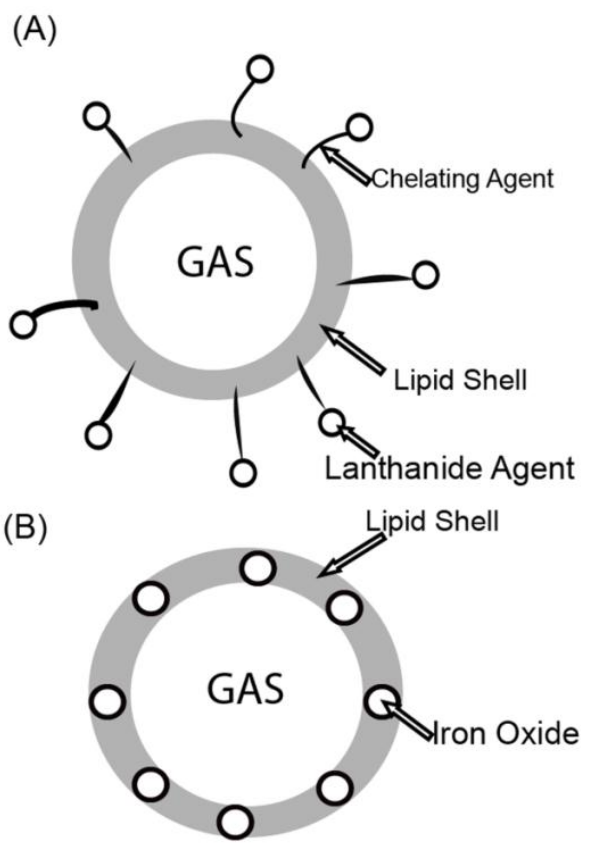

to microvasculature more efficiently than nonmagnetic microbubbles, which make magnetic microbubbles a good alternative to nonmagnetic microbubbles for vascular molecular imaging [28-30].

\section{Preparation of magnetic microbubbles}

Gas-filled and polymer/lipid/surfactant encapsulated microbubbles have been well established in the last 20-30 years. They can be prepared by various methods, such as sonication [31-33], high shear emulsification [34, 35], membrane emulsification [36, 37], ink jet printing [38], electrohydrodynamic atomization [39, 40] and microfluidic processing [41-44].

Stride and Edirisinghe [14] summarized the traditional and newly emerging techniques to prepare microbubbles and compared the differences of these techniques. The preparation of magnetic microbubbles is similar to microbubble preparation methods but with the addition of magnetic nanoparticles. Fig. 1 shows the different microbubble constructs with magnetic nanoparticles [45]. Fig. 1(A) shows the construct that magnetic nanoparticles are coupled to the shell surface of the microbubble through certain chelating agent or electrostatic coupling. Fig. 1(B) shows the structure that iron oxide nanoparticles are embedded in the microbubble shell. And in Fig. 1(C) nanoparticles are embedded in the oil layer of the microbubble.

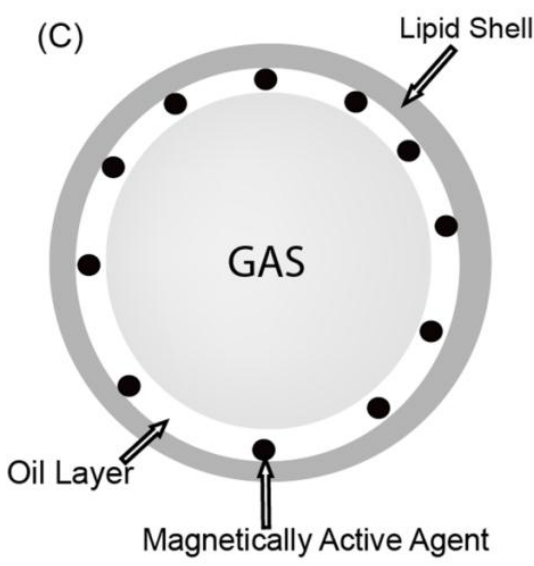

Figure I. Schematic diagram of different magnetic microbubble constructs [45]. A. magnetic nanoparticles are coupled to the shell surface of the microbubble. B. iron oxide nanoparticles are embedded in the microbubble shell. C. nanoparticles are embedded in the oil layer of the microbubble. 
A

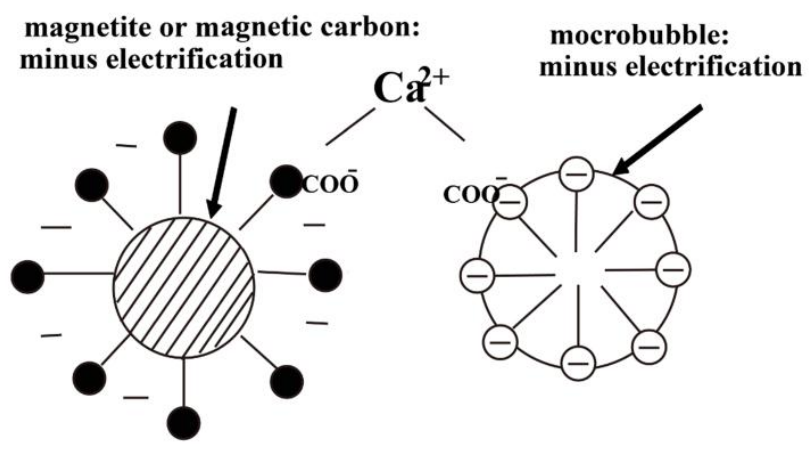

B

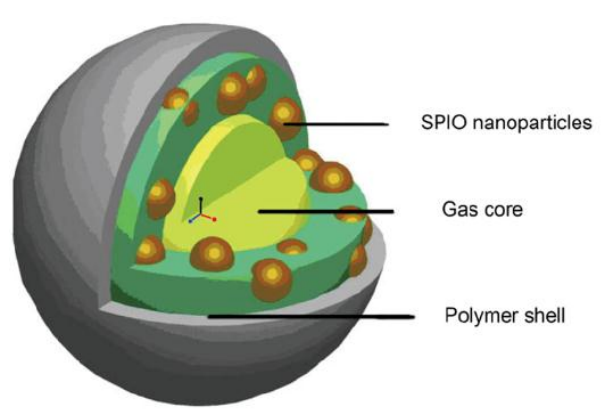

Figure 2. Schematics of two different magnetic microbubbles [25, 23]

Fig. 2(A) shows an entity of Fig. 1(A) generated by electrostatic coupling [25]. Soetanto et al. covered magnetite particles with an anionic surfactant to make them negatively charged and their self-made surfactant microbubbles were also negatively charged. In the end they use calcium ions as multivalent anions to connect the magnetites to the surface of the surfactant microbubbles. Actually, in order to obtain magnetic microbubbles with nanoparticles coupled to the surface of the shell, one can also use microfluidic devices, the coupling process is much easier [13]. For example, Park et al. mixed an aqueous solution containing anionic $\mathrm{Fe}_{3} \mathrm{O}_{4}$ nanoparticles, lysozyme and alginate with $\mathrm{CO}_{2}$ gas to the three channels through syringe pumps to form stable functionalized magnetic microbubbles in a narrow size distribution (Fig. 3(A)).To entrap magnetic nanoparticles into oil layer (inner layer) of a double-layered microbbuble (Fig. 1(C)), Yang et al. [23] used a double solvent evaporation interfacial deposition process (double-emulsion procedure) to prepare magnetic microbubbles which have a polymer shell with superparamagnetic iron oxide (SPIO) nanoparticles embedded inside (Fig. 2(B)). The prepared magnetic microbubbles have a mean diameter of $3.98 \mu \mathrm{m}$ with polydispersity index of 0.425 . Chow et al. [27] used a similar method (Fig. 3(C)) to prepare MION-entrapped PMBs (monocrystalline iron oxide nanoparticles-entrapped polymer microbubbles).

Liu et al. [17] used one-pot emulsion polymerization to embed iron oxide nanoparticles into the microbubble shells (Fig. 3(B)), this is a living example as showed in Fig. 1(B). They used a syringe to add butyl cyanoacrylate (BCA) into freshly prepared ultrasmall superparamagnetic iron oxide (USPIO) solution, and then agitated the mixture at a high speed (1000 rpm) at room temperature. Then they collected the upper solid foam after 4 days. At last they washed out the impurities with Triton X-100 and centrifuged the
UPMB (USPIO-PBCA microbubble, that is ultrasmall superparamagnetic iron oxide-poly(butyl cyanoacrylate) microbubble) suspension to collect the supernatant bubble-cake. After ultrasound exposure, the iron oxide nanoparticles inside the microbubble shells are released out.

In a word, it's hard to say which one of the present methods is the best to prepare stable, monodispersed magnetic microbubbles because each method has its advantages and disadvantages respectively. Based on the present methods, in order to obtain the better size distribution, the cost of the processing inevitably would be increased because of the low yield. Therefore, specific preparation methods are employed to meet corresponding needs. And the development of novel preparation methods is also still attracting in the future research.

\section{Magnetic microbubbles as imaging con- trast agents (diagnostic)}

\section{I. Dual modality imaging contrast agents}

Although ultrasound imaging is a safe and non-invasive imaging technique in the diagnostic field, the signal is usually disturbed by many factors. The ability of ultrasound to reveal the tissue structure is worse than that of CT and MRI. So it's a good option to combine US imaging with other imaging modalities, such as MRI. Magnetic microbubbles can make this formulation come true. The magnetic nanoparticles embedded in the bubbles can be excellent contrast agents for MRI. On the other hand, the microbubbles are proven to be good contrast agents for ultrasound imaging. Furthermore, gas-filled microbubbles can potentially be used as an intravascular MR susceptibility contrast agent in vivo because of the induction of large local magnetic susceptibility difference by the gas-liquid interface. The dual-mode imaging could offer more valuable information to 
make more accurate diagnosis than either modality alone. In MRI, the magnetic field can also guide the magnetic microbubbles to the specific locations, making the imaging more efficient.
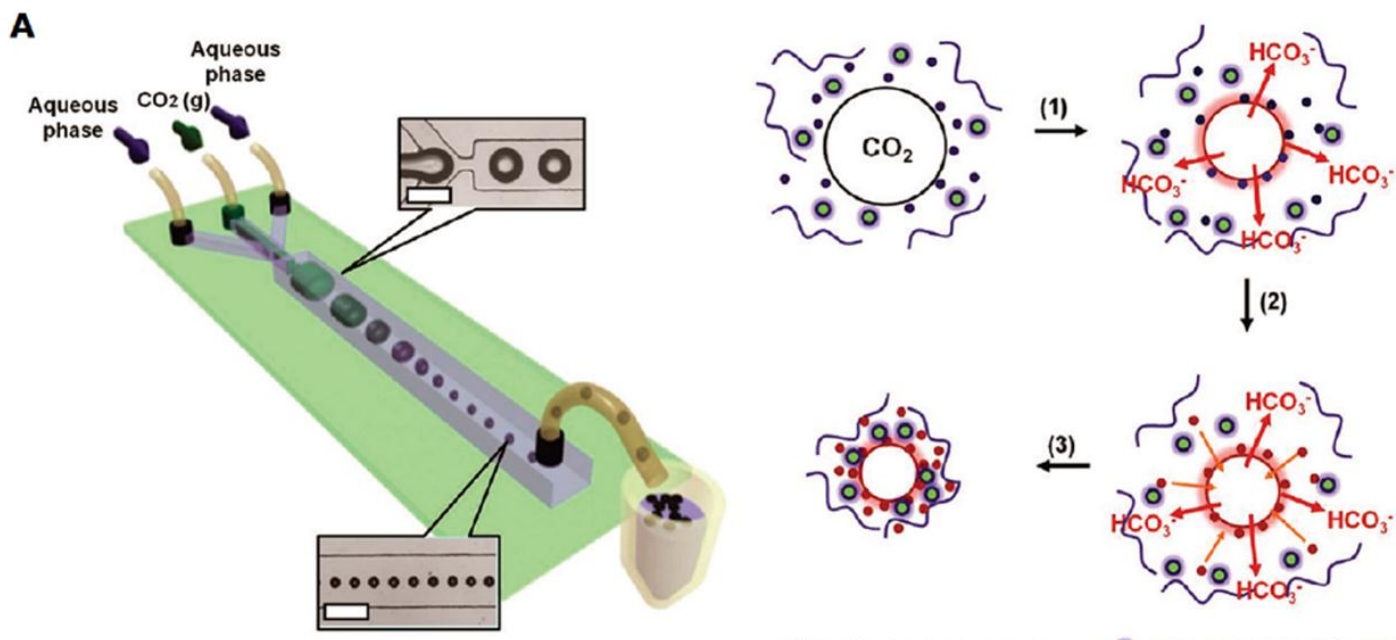

B
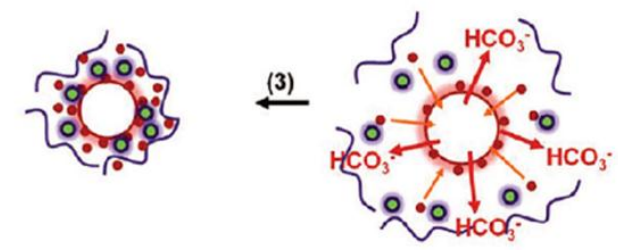

- Negatively charged lysozyme Anionic nanoparticle (NP)

- Positively charged lysozyme $\sim$ Alginate

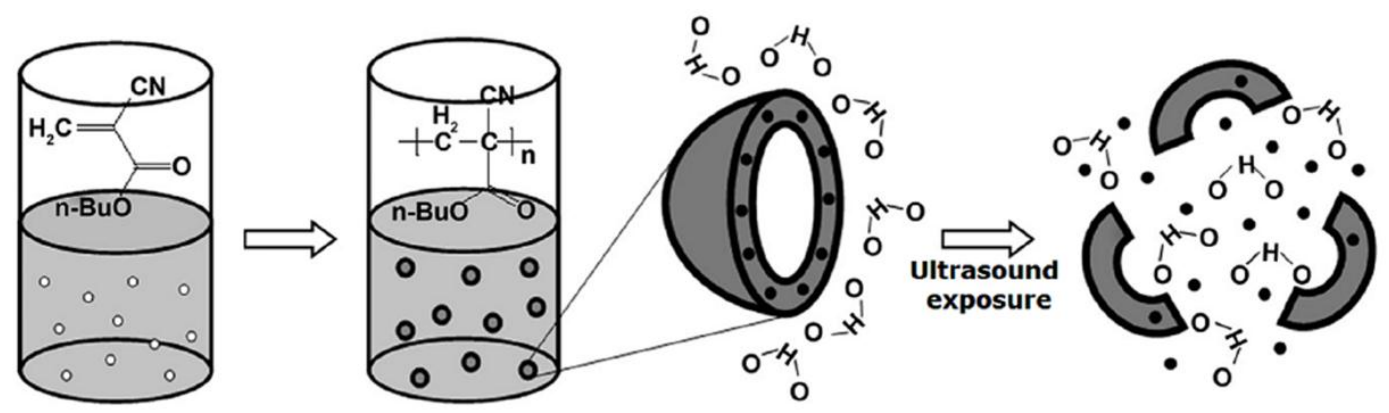

PLGA: Poly (D,L-lactide-co-glycolic acid) 50:50

C

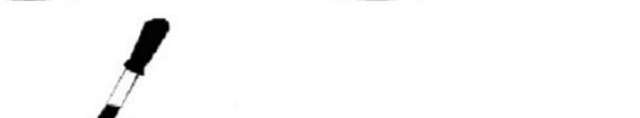
MION: Monocrystalline iron oxide nanoparticles PVA: Poly (vinyl alcohol)
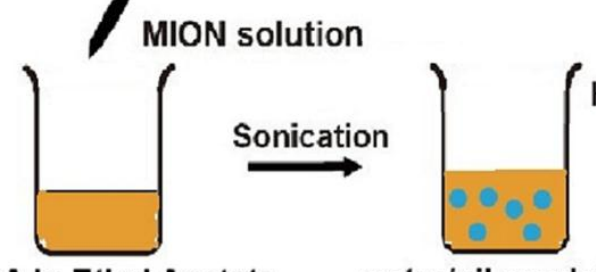

waterioil emulsion

(water/oil)/water emulsion

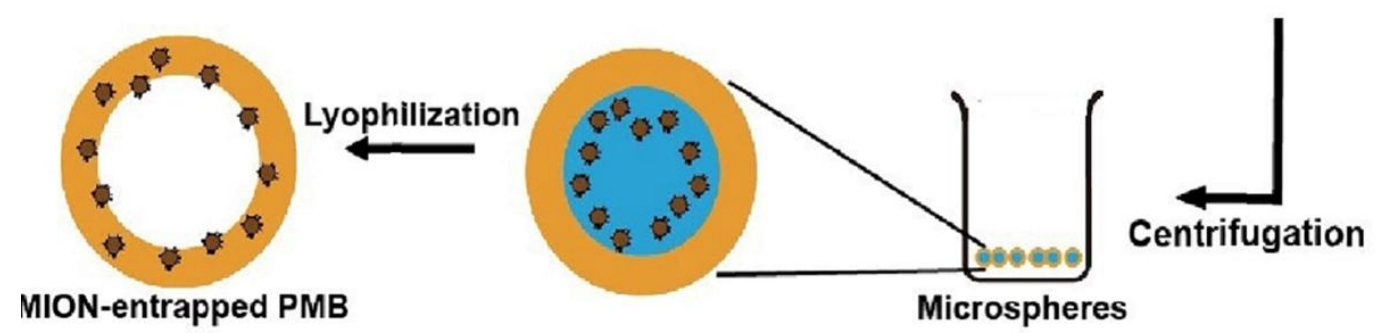

Figure 3. Schematic illustrations of three different procedures $[13,17,27]$. A: micro fluidic device: membrane materials, nanoparticle solution and the gas are pumped into the micro tubes to form magnetic microbubbles through electrostatic coupling; B: one-pot emulsion polymerization: consisting of the emulsion polymerization of BCA and the encapsulation of USPIO; C: double-emulsion: consisting of the forming of a water/oil emulsion and (water/oil)/water emulsion, and lyophilization to entrap MION into the oil layer of the bubble shell. 
The magnetic microbubbles that Yang et al. [23] prepared are $\mathrm{Fe}_{3} \mathrm{O}_{4}$-inclusion PLA-PVA (poly (DL-lactide)-polyvinyl alcohol) double-layered microbubbles. They embedded various concentrations of $\mathrm{Fe}_{3} \mathrm{O}_{4}$ nanoparticles in the oil layer of the constant shell. Compared with non-SPIO-inclusion microbubbles, the transverse relaxation rate $\left(R_{2}\right)$ of SPIO-inclusion microbubbles increases more rapidly with the increase of microbubble volume fraction. When the microbubble volume fraction reaches greater than $60 \%$, the SPIO-inclusion EMB (encapsulated microbubble) solution can enhance transverse relaxivity significantly. This result indicates that SPIO-inclusion EMBs can act better in MRI compared with non-SPIO-inclusion microbubbles. As for ultrasound imaging, SPIO-inclusion microbubbles enhance the image contrast significantly compared with non-SPIO-inclusion microbubbles. On the other hand, with the increase of the inclusion concentration, the enhance ability of SPIO-inclusion EMBs increased first and decreased later, animal experiments verified the results.

Similar phenomena were observed in the case of Liu's research [17] in which the USPIOs were entrapped in the hard shell of the microbubble. They used US-MR phantom imaging assay to determine the acoustic and magnetic properties of the microbubbles. Compared with pure microbubbles, UPMBs (USPIO-PBCA microbubbles) generated comparable signals both in ultrasound imaging and MRI. When the magnetic nanoparticles are coupled to the surface of the microbubble [13], they can also increase the $\mathrm{T}_{2}$ relaxation rate of the water near the ROI (region of interest) to enhance the negative signal.

The above results indicate that no matter the magnetic nanoparticles are coupling to the surface of the microbubble shell, entrapped in the shell or embedded in the inner layer of the microbubble shell, the magnetic microbubbles can highly enhance both ultrasound imaging contrast and MRI contrast [3, 13, 17].

Of all the three described examples, when the concentration of the magnetic nanoparticles rises, the enhancing ability of magnetic microbubbles increases first and then decreases in MRI, and the extremely high embedded magnetic nanoparticle concentration results in relatively low ultrasound contrast intensity. The explanation to the phenomenon is that the extremely high concentration embedded magnetic nanoparticles could hinder the oscillation of the microbubbles. According to the above research results, we can choose moderate magnetic nanoparticle concentration of magnetic microbubbles to gain the best US \& MR imaging efficacy, for example in the refer- ence 23 , the moderate concentration may be set at $80 \%$.

Furthermore, microbubbles can potentially be used as an intravascular MR susceptibility contrast agent in vivo because of the induction of large local magnetic susceptibility difference by the gas-liquid interface [26]. The magnetic susceptibility of the microbubbles locally perturb the static magnetic field, which influences the transverse-relaxation properties of the surrounding medium. Changes in the pressure after the bubble dimensions affect the magnetic field perturbations and subsequently the transverse relaxation. But compared with other intravascular MR susceptibility contrast agents, the susceptibility effect of microbubbles is relatively weak. Chow et al. [27] found that by entrapping MIONs into PMB shells could enhance the microbubble susceptibility, hence, the magnetic microbubbles can serve as a good intravascular MR contrast agent and blood pressure sensor.

But here comes a problem: How to form a kind of magnetic microbubbles to gain the strongest intravascular MR susceptibility? Dharmakumar et al. [45] resolved this problem through analytical approximations and numerical simulations. They used finite-element simulations to demonstrate that the effective volumetric magnetic susceptibility of a coated microbubble is dependent on the radius, the shell volume fraction and the magnetic susceptibility of the particulates on the shell. The results suggest that a suitable microbubble formulation which will be MR-sensitive to small pressure changes at $1.5 \mathrm{~T}$ must be $2-3 \mu \mathrm{m}$ in radius and be uniformly coated with single-domain magnetic nanoparticles, and the shell volume fractions should be below $5 \%$, which means the magnetic microbubble shells should be very thin. Actually most lipid-encapsulated and surfactant-encapsulated microbubbles are up to the mustard.

In a word, dual modality imaging for ultrasound and magnetic resonance imaging is the main application of magnetic microbubbles. The key factors that influence the dual modality imaging quality of magnetic microbubbles are the stability of micobubbles and the viscosity of the bubble membrane. To obtain images of good quality, we should choose proper membrane materials (polymers, lipids or surfactants) and moderate magnetic nanoparticle concentration to form magnetic microbubbles for better clinical applications.

\subsection{Molecular imaging contrast agents}

Angiogenesis is the normal physiological process for newblood vessels to develop from preexisting 
ones, it is a keystone in the treatment of cancer and potentially many other diseases, such as cardiovascular diseases. With the introduction of microbubbles that can be targeted to the molecular markers related to angiogenesis (VEGFR-2, integrin $\alpha_{v} \beta_{3}$, and endoglin), ultrasound imaging has become an attractive imaging modality to non-invasively assess tumor angiogenesis.

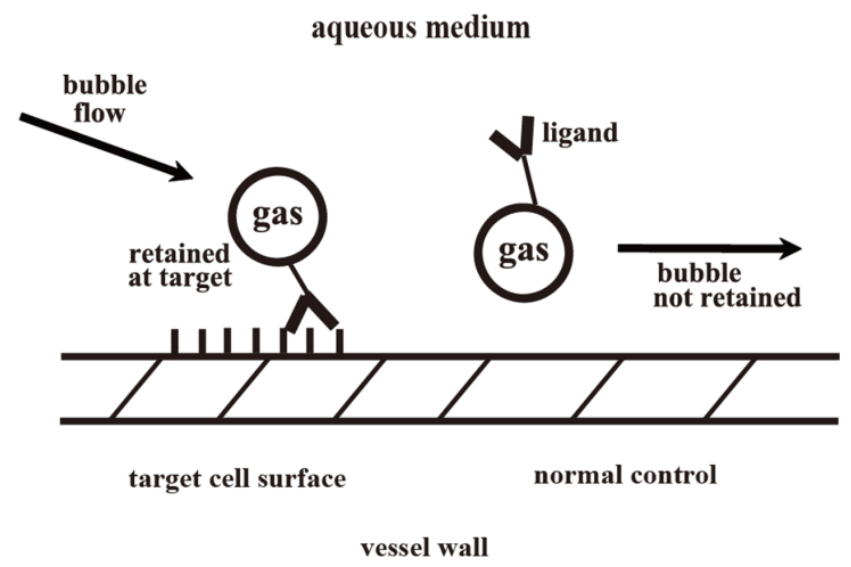

Figure 4. The schematic of molecular imaging with targeted ultrasound contrast agents [47].
Molecular imaging with targeted ultrasound contrast agents (Fig. 4) can be illustrated as the following: A specific ligand, which can bind to the receptor of interest, is attached to the shell of a gas-filled microbubble. The resulting targeted contrast material circulates for a period of time, and is gradually cleared. At the same time, targeting occurs due to the specific ligand-receptor interaction, and some of the microbubbles which had a chance to flow through the target area are selectively attached to the receptor. The area with high level contrast marks the receptor/disease site and can be imaged [47].

However, traditional imaging methods act well in atherosclerosis, angiogenesis and inflammation of microvasculature but not larger vessels (such as aorta) due to the high flow speed of the blood in larger vessels (high shear stress) [4-5]. Molecular imaging with magnetic microbubbles may resolve this difficulty: with the guidance of the magnetic field, magnetic microbubbles can accumulate in the region of interest, the targeting molecular markers then can specifically attach to the tissue or cells to offer a real-time, noninvasive visualization at the molecular level.To make it possible to enhance the ultrasound molecular imaging efficiency of atherosclerosis in the aorta, Wu et al. [28] prepared magnetic microbubbles conjugated with anti-VCAM (vascular cell adhesion molecule)-1 antibodies (Fig. 5) to enhance the molecular imaging efficiency of atherosclerosis.

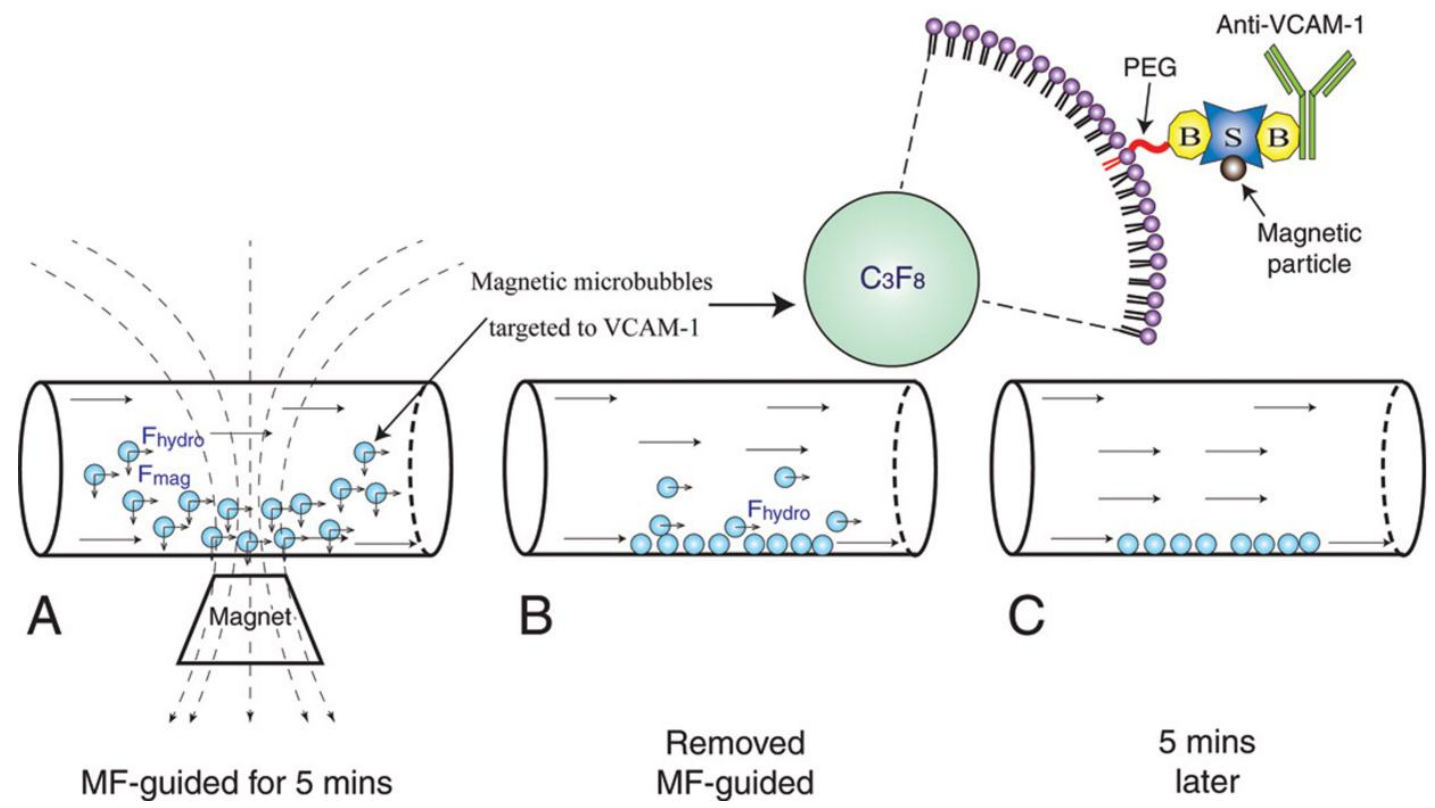

Figure 5. Illustration of microbubble axial flow behavior and magnetic field-guided targeting [28]. 
This kind of magnetic microbubbles can easily target to VACM-1, with the introduction of magnetic field, the microbubbles can accumulate in the specific vascular areas.

Three kinds of microbubbles are made: the magnetic VCAM-1-targeted microbubbles (MVMBs), the untargeted magnetic microbubbles (MMBs) and the nonmagnetic VCAM-1-targeted microbubbles (VMBs). The results showed that MVMBs attached to the VCAM-1-Fc (Fc section of anti-VCAM-1 antibody) coated plates just like VMBs in the absence of magnetic field guidance, but when the magnetic field was introduced, attachment of MVMBs was significantly higher than that of VMBs. Animal experiments showed outstanding attachment to aorta of the magnetic microbubbles and the microbubbles were distributed throughout the aorta. US imaging of atherosclerosis guided by magnetic field showed significant diversity. The mean video intensity of MVMBs was the strongest, followed by the VMBs, the lowest video intensity was for the MMBs. After 5-minute flush, the three kinds of microbubbles in wild-type mice (healthy mice without atherosclerosis) showed similar video intensity, while the magnetic microbubbles of experimental sets (apolipoprotein E-deficient mice) still remained significant video intensity.

This result means the anti-VCAM-1 antibody coated magnetic microbubbles can efficiently attach to the inner wall of aorta even at a very high shear stress, resulting in improved detection of the early stages of atherosclerosis, which makes them excellent atherosclerosis imaging contrast agents. They also used biotinylated anti-mice P-selectin (MBpm) to replace the anti-VCAM-1 antibody to form a similar type of microbubbles which can attach to endothelial P-selectin, making the magnetic microbubbles to be excellent magnetic guided inflammatory molecular imaging contrast agents in high-shear flow [29].

The main advantage of antibodies modified magnetic microbubbles to be vascular molecular imaging contrast agents is that with the magnetic field guiding to the specific location and the binding of specific molecules to the endothelial cells, the double attraction forces (the magnetic force and the binding force of the coupled molecules) make the magnetic microbubbles attached to the vascular firmly even in a high shear stress, while the situation of non-magnetic microbubbles things is quite different.

\section{Magnetic microbubbles for drug delivery (therapeutic)}

To minimize drug degradation and loss, to prevent harmful side-effects and to increase drug bioavailability and the fraction of the drug accumulated in the required zone, various drug delivery and drug targeting systems are currently developed or under development [48-50].

\section{I. Drug delivery systems}

Microbubble can be a good type of drug delivery system due to the phenomenon called sonoporation: Cell membranes can become temporarily "open" to small and large molecules and then "resealed" when relatively low amplitude ultrasound is applied to a cell suspension that contains an ultrasound contrast agent. Consequently, foreign molecules may be trapped in the cells. [6-8]. If we trap drugs inside the microbubbles, with the sonopoartion, the drugs will be released out of the microbubbles, and then swallowed by the temporally opened cells and trapped inside them. Magnetic microbubbles are especially outstanding drug delivery systems of microbubble-type drug delivery systems because they can be guided by the magnetic field to the specific locations of interest.

$\mathrm{Fe}_{3} \mathrm{O}_{4}$-inclusion microbubbles can be used as in situ drug delivery systems other than dual-modality contrast agents for ultrasound imaging and MRI [24]. When a focused ultrasound is introduced to a cell solution with the presence of $\mathrm{Fe}_{3} \mathrm{O}_{4}$-inclusion microbubbles, sonoporation occurs. Then the microbubbles can be destroyed, consequently the embedded $\mathrm{Fe}_{3} \mathrm{O}_{4}$ nanoparticles will be released from the shells of the microbubbles, at this moment the transiently opened cells can adopt the nanoparticles, which will result in a series of bioeffects on the cells. Yang et al. studied the delivery efficiency of the $\mathrm{Fe}_{3} \mathrm{O}_{4}$ nanoparticles and other bioeffects. They found that the $\mathrm{Fe}_{3} \mathrm{O}_{4}$ nanoparticles embedded in the shells of microbubbles can be delivered into the tumor cells, and the delivery rate increases as the acoustic intensity increases. The entered $\mathrm{Fe}_{3} \mathrm{O}_{4}$ nanoparticles possessed a suitable cytotoxicity, the cell apoptosis increases as the acoustic intensity increases. Compared with non- $\mathrm{Fe}_{3} \mathrm{O}_{4}$-inclusion microbubbles, the $\mathrm{Fe}_{3} \mathrm{O}_{4}$ embedded microbubbles have lower bioeffects because the embedded nanoparticles enhance the stability of the microbubbles, and subsequently hinder the oscillation of the microbubbles.

When the $\mathrm{Fe}_{3} \mathrm{O}_{4}$ nanoparticles are delivered into cells, they can cause an effect called hyperthermia with an alternating magnetic field introduced, causing corresponding cell damage. Although this work is not strictly a drug delivery case as it only delivers the nanoparticles inside the bubble shell to tumor cells, not the specific drugs, the $\mathrm{Fe}_{3} \mathrm{O}_{4}$ nanoparticles can serve as hyperthermia agents in some conditions. We can also predict that if we filled the $\mathrm{Fe}_{3} \mathrm{O}_{4}$-inclusion 
microbubbles with specific drugs, for example, doxorubicin (a tumor suppression drug), we can effectively delivery the drug to tumor tissue with magnetic field guiding.

\subsection{Gene transfection systems}

If we replace drugs with nucleic acids, drug delivery systems described above can become gene transfection systems. Of many gene transfection models, magnetic targeting and ultrasound -enhanced delivery is gaining more and more attention.

Vlaskou et al. [51] formed magnetic microbubbles through self-assembly of the lipids, magnetic nanoparticles and nucleic acids (Fig. 6). They used three different types of nucleic acids (the luciferase reporter gene in NIH3T3 mouse fibroblasts, in H441 human lung epithelial cells and luciferase siRNA) to study the delivery efficiency in cultured adherent cells, the results showed that when a magnetic field was applied, efficient delivery of these nucleic acids' delivery was achieved and it was comparable with commercially available transfection reagents (SONOVUE microbubbles). And the transfection effi- ciency was much higher than when using plasmid applied with SONOVUE microbubbles (one kind of commercially available lipid microbubbles) and ultrasound. Combined use of ultrasound and the magnetic field didn't further enhance the transfection efficiency. The in vivo experiments in mice showed that when a magnetic field was introduced to the mouse chest, the accumulation of plasmid DNA in the targeted site would be two to three fold of the untargeted site in the lungs. They used the similar formulation to transfer a report gene to HeLa cells, the cells treated with the microbubbles and ultrasound expressed a dose-dependent cytotoxicity. This formulation of magnetic microbubbles can be a promising gene delivery vector and cancer gene therapy reagent.

Since the genes can be coupled or entrapped to the magnetic microbubbles, the gene delivery process becomes controllable and visible by the guidance of the dual-modality imaging. Based on the magnetic microbubble, the gene delivery system may have more promising applications.
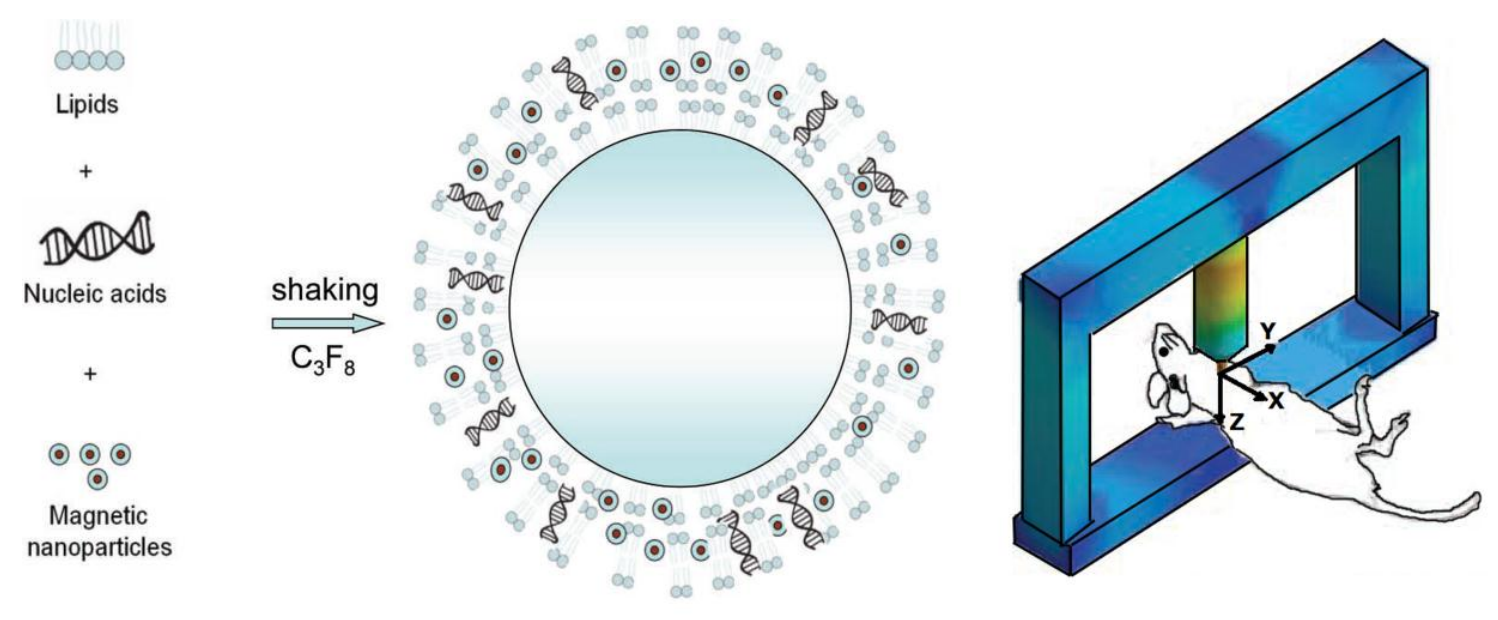

Figure 6. Schematic of the self-assembly of the lipids, MNPs, and nucleic acids into magnetic microbubbles and the animal experiment setting [5I].

\section{Conclusion and perspective}

Although the magnetic microbubbles play an important role in preclinical diagnostic and therapeutic fields due to their excellent properties to be dual modal contrast agents for US imaging and MRI, drug delivery systems, nucleic acid delivery systems and molecular imaging contrast agents, present studies are still limited to simulated researches, cell researches and small animal studies [30]. Future work of magnetic microbubbles could be focused on the de- velopment of multi-modal imaging contrast agents, accurate administration of drug/gene delivery system and clinical translation.

\section{Acknowledgements}

This work was supported by the National Important Science Research Program of China (Nos. 2011CB933500), National Natural Science Foundation of China (Nos. 50872021, 31000453), the Basic Research Program of Jiangsu Province (Natural Science Foundation, No. BK2009013) and Research Fund of 
Young Teachers for the Doctoral Program of Higher Education of China (Nos. 20100092120038).

\section{Conflict of Interest}

The authors have declared that no conflict of interest exists.

\section{References}

1. Ke H, Wang J, Dai Z, et al. Gold-nanoshelled microcapsules: a theranostic agent for ultrasound contrast imaging and photothermal therapy. Angew Chem Int Ed 2011; 123: 3073-7.

2. Wink MH, Wijkstra H, Rosette JJ, Grimbergen CA. Ultrasound imaging and contrast agents: A safe alternative to MRI? Minim Invasive Ther 2006; 15(2): 93-100.

3. Yang F, Li L, Li Y, et al. Superparamagnetic nanoparticle-inclusion microbubbles for ultrasound contrast agents. Phys Med Biol 2008; 53: 6129-41.

4. Lindner JR, Song J, Jayaweera AR, Sklenar J, Kaul S. Microvascular rheology of definity microbubbles after intra-arterial and intravenous administration. J Am Soc Echocardiogr 2002; 15(5): 396-403.

5. Ismail S, Jayaweera AR , Camarano G, Gimple LW , Powers ER , Kaul S. Relation between air-filled albumin microbubble and red blood cell rheology in the human myocardium: influence of echocardiographic systems and chest wall attenuation. Circulation 1996; 94 (3): 445-51.

6. Wu J, Ross JP, Chiu JF. Reparable sonoporation generated by microstreaming. J Acoust Soc Am 2002; 111: 1460-4.

7. Yang F, Gu N, Chen D, et al. Experimental study on cell self-sealing during sonoporation. J Control Release 2008; 131: 205-10.

8. Lentacker I, Geers B, Demeester J, et al. Design and evaluation of doxorubicin-containing microbubbles for ultrasound-triggered doxorubicin delivery: cytotoxicity and mechanisms involved. Mol Ther.2010; 18: 101-8.

9. Ohta S, Suzuki K, Ogino K, et al. Gene transduction by sonoporation. Develop Grouth Differ 2008; 50: 517-20.

10. Deckers R, Moonen CT. Ultrasound triggered, image guided, local drug delivery. J Controlled Release 2010; 148: 25-33.

11. Larina IV, Evers BM, Esenaliev RO. Optimal drug and gene delivery in cancer cells by ultrasound-induced cavitation. Anticancer Res 2005; 25: 149-56.

12. Reslan L, Mestas JL, Herveau S, et al. Transfection of cells in suspension by ultrasound cavitation. J Control Release 2010; 142: 251-8.

13. Park JI, Jagadeensan D, Williams R, et al. Microbubbles loaded with nanoparticles: a route to multiple imaging modalities. ACS Nano 2010; 4: 6579-86.

14. Stride E, Edirisinghe M. Novel microbubble preparation technologies. Soft matter 2008; 4: 2350-9.

15. Zhang Y, Yang Y, Cai W. Multimodality imaging of integrin $\mathrm{a}_{\mathrm{v}} \beta_{3}$ expression. Theranostics 2011; 1: 135-48.

16. Kiessling F, Gaetjens J, Palmowski M. Application of molecular ultrasound for imaging integrin expression. Theranostics 2011; 1: 127-34.

17. Liu Z, Lammers $\mathrm{T}$, Ehling $\mathrm{J}$, et al. Iron oxide nanoparticle-containing microbubble composites as contrast agents for MR and ultrasound dual-modality imaging. Biomaterials 2011; 32: 6155-63.

18. Laurent S, Forge D, Port M, et al. Magnetic iron oxide nanoparticles: Synthesis, Stabilization, vectorization, physicochemical characterizations, and biological applications. Chem Rev 2008; 108: 2064-110.
19. Gupta AK, Gupta M. Synthesis and surface engineering of iron oxide nanoparticles for biomedical applications. Biomaterials 2005; 26: 3995-4021.

20. Konczol M, Ebeling S, Goldenberg E, et al. Cytotoxicity and genotoxicity of size-fractionated iron oxide (magnetite) in A549 human lung epithelial cells: role of ROS, JNK, and NF-K.B. Chem Res Toxicol 2011; 24: 1460-75.

21. Silva AC, Oliveira TR, Mamani JB, et al. Application of hyperthermia induced by superparamagnetic iron oxide nanoparticles in glioma treatment. Int J Nanomed 2011; 6: 591-603.

22. Yu M, Park J, Jon J. Targeting strategies for multifunctional nanoparticles in cancer imaging and therapy. Theranostics 2012; 2: 3-44.

23. Yang F, Li Y, Chen Z, et al. Superparamagnetic iron oxide nanoparticle-embedded encapsulated microbubbles as dual contrast agents of magnetic resonance and ultrasound imaging. Biomaterials 2009; 30: 3882-90.

24. Yang F, Zhang M, He W, et al. Controlled release of $\mathrm{Fe}_{3} \mathrm{O}_{4}$ nanoparticles in encapsulated microbubbles to tumor cells via sonoporation and associated cellular bioeffects. Small 2011; 7: 902-10.

25. Soetanto K, Watarai H. Development of magnetic microbubbles for drug delivery system (DDS). Jpn J Appl Phys 2000; 39: 3230-2.

26. Wong KK, Huang I, Kim YR, et al. In vivo study of microbubbles as an MR susceptibility contrast agent. Magn Reson Med 2004; 52: $445-52$.

27. Chow AM, Chan WY, Cheung JS, Wu EX. Enhancement of gas-filled microbubble R2* by iron oxide nanoparticles for MRI. Magn Reson Med 2010; 63: 224-9.

28. Wu J, Poi HL, Bin J, et al. Efficacy of contrast-enhanced US and magnetic microbubbles targeted to vascular cell adhesion molecule-1 foe molecular imaging of atherosclerosis. Radiology 2011; 260: 463-71.

29. Bin J, Wu J, Yang $\mathrm{L}$, et al. In vivo assessment of novel magnetic microbubbles targeted to P-selectin for inflammatory molecular imaging in high-shear flow. Circulation. 2009; 120: S327.

30. Nahrendorf $M$, Sosnovik DE. Science to practice: will magnetic guidance of microbubbles play a role in clinical molecular imaging? Radiology 2011; 260: 309-10.

31. Unger E, McCreery T, Sweitzer R, et al. Acoustically active lipospheres containing paclitaxel - A new therapeutic ultrasound contrast agent. Invest Radiol 1998; 33: 886-92.

32. Zhao $Y$, Liang H, Mei X, Halliwell M. Preparation, characterization and in vivo observation of phospholipid-based gasfilled microbubbles containing hirudin. Ultrasound Med Biol 2005; 31: 1237-43

33. Christiansen C, Kryvi H, Sontum P, Skotland T. Physical and biochemical-characterization of albunex (TM): A new ultrasound contrast agent consisting of air-filled albumin microspheres suspended in a solution of human albumin. Biotechnol Appl Biochem 1994; 19: 307-20.

34. Bjerknes K, Dyrstad K, Smistad G, Agerkvist I. Preparation of polymeric microcapsules: Formulation studies. Drug Dev Ind Pharm 2000; 26: 847-56.

35. Jiang B, Gao C, Shen J. Polylactide hollow spheres fabricated by interfacial polymerization in an oil-in-water emulsion system. Colloid Polym Sci 2006; 284: 513-9.

36. Bao D, Zhang H, Liu X, Zhao Y, Ma X, and Yuan Q. Preparation of monodispersed polymer microspheres by SPG membrane emulsification-solvent evaporation technology. J Dispersion Sci Technol 2007; 28: 485-90.

37. Kukizakia M, Goto M. Spontaneous formation behavior of uniform-sized microbubbles from Shirasu porous glass (SPG) membranes in the absence of water-phase flow. Colloids Surf A 2007; 296: 174-81. 
38. Bohmer M, Schroeders R, Steenbackkers J, et al. Preparation of monodisperse polymer particles and capsules by ink-jet printing. Colloids Surf A 2006; 289: 96-104.

39. Farook U, Zhang HB, Edirisinghe M, et al. Preparation of microbubble suspensions by co-axial electrohydrodynamic atomization. Med Eng Phys 2007; 29: 749-54.

40. Farook U, Stride E, Edirisinghe M, Moaleji R. Microbubbling by co-axial electrohydrodynamic atomization. Med Biol Eng Comput 2007; 45: 781-9.

41. Whitesides G. The origins and the future of microfluidics. Nature 2006; 442: 368-73.

42. Xu Q and Nakajima M. The generation of highly monodisperse droplets through the breakup of hydrodynamically focused microthread in a microfluidic device. Appl Phys Lett 2004; 85: 3726-8.

43. Garstecki P, Gitlin I, DiLuzio W, et al. Formation of monodisperse bubbles in a microfluidic flow-focusing device. Appl Phys Lett 2004; 85: 2649-51.

44. Pancholi K, Farook U, Moaleji R, et al. Novel methods of preparing phospholipid coated microbubbles. Eur Biophys J 2008; 37: 515-20.

45. Dharmakumar R, Plewes DB, Wright GA. A novel microbubble construct for intracardiac or intravascular MR manometry: a theoretical study. Phys Med Biol 2005; 50:4745-62.

46. Tu J, Guan J, Qiu Y, Matula TJ. Estimating the shell parameters of SonoVue ${ }^{\circledR}$ microbubbles using light scattering. J Acoust Soc Am 2009; 126 (6): 2954-9.

47. Klibanov AL. Ultrasound molecular imaging with targeted microbubble contrast agents. J Nucl Cardiol 2007; 14: 876-84.

48. Jeong B, Bae YH, Lee DS, Kim SW. Biodegradable block copolymers as injectable drug-delivery systems. Nature 1997; 388: 860-2.

49. Allen TM, Cullis PR. Drug delivery systems: entering the mainstream. Science 2004; 303: 1818-22.

50. Torchilin VP. Structure and design of polymeric surfactant-based drug delivery systems. J Control Release 2001; 73: 137-72.

51. Vlaskou D, Mykhaylyk O, Krotz F, et al. Magnetic and acoustically active lipospheres for magnetically targeted nucleic acid delivery. Adv Funct Mater 2010; 20: 3881-94. 